\section{MS10-P6 Inside "false tobacco"}

Agata E. Owczarzak ${ }^{1}$, Maciej Kubicki ${ }^{1}$
Keywords: cytisine, charge distribution, electron density, topological analysis, hydrogen bond, weak interactions

1. Faculty of Chemistry, Adam Mickiewicz University in Poznan, Poland

email: agataowczarzak@gmail.com

Tobacco smoking kills around 6 million people each year and it's one of the main public health threats. Smoking cessation with Cytisine is healthy and very effective. Cytisine is natural alkaloid obtained from Laburnum anagyroides, during the Second World War the levels of this plants were called "false tobacco". It's a partial agonist at the $\alpha 4 \beta 2$ nAChR (nicotinic acetylcholine receptors) and plays the part of nicotine substitute. Nicotine and cytisine have similar a mechanism action, but cytisine substance has low toxicity in contrast to nicotine. Therefore, cytisine has been applied in nicotine replacement therapy in the form of Tabex ${ }^{\circledR}$ Desmoxan ${ }^{\circledR}$ Chantix ${ }^{\circledR}$ etc. Moreover, cytisine derivatives have been explored as potential drugs Alzheimer's and Parkinson's diseases[1].It is important to note that still, a thorough understanding of structural requirements of $\alpha 4 \beta 2$ agonists is lacking. High resolution $\mathrm{X}$-ray crystallography can be used as main tool in analysis of structure relationships-activity. In this work will be present the analyse charge density distribution and intermolecular interactions in the cytisine and some of its new derivatives. The structures have been refined using Hansen-Coppens multipolar model [2] implemented in MoPro software [3], using several different strategies to obtain the best model of crystals. We will concentred on a comparison of the multipole parameters and topological analyses in free base, protonated form and N-substituted cytisine.

This work is supported bya grant from the Polish National Science Center,2013/11/B/ST5/01681

[1] P. Tutka, W. Zatonski , Pharmacological Reports 58, 777-798( 2005).

[2] N. Hansen, P. Coppens, Acta Crystallographica Section A 34, 909-921 (1978).

[3] C. Jelsch, B.Guillot, A. Lagoutte, C. Lecomte, J. Appl. Cryst. 38,38-54 (2005).

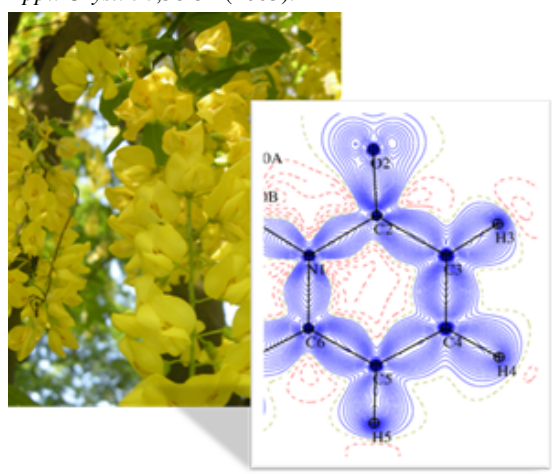

Figure 1. Schematic illustration: Laburnum anagyroides "false tobacco" and static deformation density map of cytisine drawn in ring $A$. 